\title{
Mobile Robots and Autonomic Ambient Assisted Living
}

DOI 10.1515/pjbr-2015-0013

Received October 15, 2014; accepted November 11, 2015

\begin{abstract}
The use of Smart Environments in the delivery of pervasive care is a research topic that has witnessed increasing interest in recent years. These environments aim to deliver pervasive care through ubiquitous sensing by monitoring the occupants Activities of Daily Living. In order for these environments to succeed in achieving their goal, it is crucial that sensors deployed in the environment perform faultlessly. In this research we investigate addressing anomalous sensor behavior through the utilization of a mobile robot. The robot's role is twofold; it must provide substitution in the presence of suspected sensor faults and act as an observer of anomalous sensor behavior in order to understand the changes that occur in the behavior of sensors deployed within the environment over time. The aim of this work is to explore a paradigm shift to the use of Autonomic Ambient Assisted Living. We have discovered that the use of a mobile robot is a viable means of introducing this paradigm to a Smart Environment.
\end{abstract}

Keywords: smart environment; mobile robots; anomalous sensors; autonomic computing; sensor substitution; ambient assisted living

\section{Introduction}

It has been estimated that approximately 2 billion people globally will be aged $60+$ by the year 2050 [1]. The increase in life expectancy is a commendable result of the advances that have been made in healthcare which are enabling us to live longer. Despite this achievement, the changing age demographics are not without their problems [2]. Whilst

\footnotetext{
^Corresponding Author: Guanitta Brady: School of Computing and Mathematics University of Ulster Jordanstown, Northern Ireland, E-mail: brady-g6@email.ulster.ac.uk

Roy Sterritt, George Wilkie: School of Computing and Mathematics University of Ulster Jordanstown, Northern Ireland, E-mail: [r.sterritt, fg.wilkie]@ulster.ac.uk
}

the number of those living longer is increasing, the amount of young people to care for them and provide support for the healthcare system is decreasing [3]. This in turn will place an even bigger strain on health service providers. As a result, there is a critical need to develop alternative means of delivering care to the older population [4].

Ambient Assisted Living (AAL) aims to address this need through the use of innovative technology to support Ageing in Place. The concept of Ageing in Place centers on the promotion of independence by enabling the elderly, especially those with cognitive impairments, to remain living in their own homes for as long as possible [5]. Several stakeholders benefit from this; care homes, care givers, those in receipt of care and their families. A reduction in the number of patients needing a place in a care home facility or regular care has the potential to benefit both care homes and care givers through the alleviation of the financial and practical strain that the provision of care to such vast quantities of elderly people will entail in the future [6].

Assistive robotics is a broad area which encompasses several different types of assistive technologies. The application of robots in intelligent environments has been shown to be beneficial in the delivery of both pervasive and social care. Some of the benefits of the use of robots in the delivery of care include [7] their inability to tire, they can be manufactured to meet demand, their increasing reliability and they are also becoming increasingly capable of delivering highly accurate data. In the care home setting robotic assistants such as Pearl the Nursebot [8] have been developed to provide support to both nursing staff and residents.

In order for the monitoring of Smart Environment occupant activities to be accurate and for occupant safety to be ensured, effective sensor functionality in the environment is essential. This means that sensing must be delivered reliably without interruption to data generation despite anomalies that may materialize in the environments' sensor technology. Sensor anomalies may manifest through irregular sensor activations stemming from sensor noise, to abnormal behavior that is a consequence of the degradation of hardware over an extended period of time. In either case, it is important that a means of ensur- 
ing the systems' ability to manage these sensor anomalies is introduced to these environments so that accurate activity detection is maintained to ultimately ensure the safety of the environments occupant. Moreover, in the event of a system being implemented and assigned the responsibility of monitoring an older person, the system is placed in a prominent role of responsibility; it has the potential to effectively remove responsibility from the human. It is therefore crucial for such a system to be dependable.

In this research we focus particularly on entry and egress in Smart Environments for those suffering from dementia. Those with dementia are often prone to forgetting the activity they are undertaking and wandering behavior [9]. This results in such occupants being particularly vulnerable. There have been several publicized instances in which those suffering from dementia have left care homes equipped with sensor technology undetected [1012]. The reasons for this vary from the sensor failing to the patient learning how to remove or deactivate the sensor. As a result of this, many of the patients in question have placed themselves in danger, with some even dying as a result of exposure to extreme weather conditions or falling victim to an accident. Such occurrences are deeply disturbing and should be preventable.

This research lays the foundations for the introduction of self-healing to a Smart Environment by establishing the viability of the utilization of a mobile robot for the purpose of introducing such functionality. In this research, we propose that robotics can be utilized to assist in enhancing the safety of vulnerable Smart Environment occupants through the investigation of anomalous static sensor behaviors and the provision of substitution for sensor faults at the point of need. In this way, the robot becomes an integral part of the Smart Environment system, which contributes to introducing self-managing capability into the environment. By introducing this AAAL [13] approach to Smart Environments utilizing robots, the system would go one step further in its responsibility for supporting its occupant by addressing its own anomalous behaviors without the need for external human intervention. It is our vision that robots may serve in a multifunctional role within the Smart Environment in order to assist in the provision of a safe, AAAL environment.

\section{Autonomic Computing}

Autonomic Computing is an approach proposed for addressing the problem of increasing computing system complexity. It was first launched in 2001 at IBM [14]. The autonomic paradigm takes its inspiration from nature; it is based on the manner in which the Autonomic Nervous System (ANS) performs its role in the body. The ANS is responsible for vital bodily functions such as the regulation of breathing, heartrate and digestive processes. The ANS performs this self-regulation without conscious thought.

In order for a system to be autonomic, it must possess four key self- ${ }^{\star}$ properties; these are referred to collectively as self-CHOP [15]. Those attributes are self-configuration, self-healing, self-optimization and self-protection. In this research we focus on the introduction of self-healing to a Smart Environment. This research is the initial step in implementing the first of the four key properties of the autonomic paradigm.

Self-healing can be exhibited in two forms according the paradigm; reactively and proactively. Reactive selfhealing is concerned with ensuring effective recovery from a fault through identifying the fault, and then, where possible, repairing it. Proactive self-healing is a process that is carried out over a longer period of time. In this form, a system monitors its behaviors in an attempt to predict and avoid reaching undesirable situations.

The goal of this research is to provide self-healing in both reactive and proactive forms. We investigate the provision of reactive self-healing through the utilization of a mobile robot as a means of investigating anomalous sensor behavior and providing sensor substitution when an anomalous sensor event occurs. We also investigate the provision of proactive self-healing through the proposed role of the robot as a watcher over time that observes the changes in static sensor behavior that occur over an extended period.

\section{Methods}

\subsection{Sensor Anomaly Types}

The first stage in this research was the establishment of the types of sensor anomalies that may be generated in a Smart Environment. In order to obtain a firm understanding of anomaly types, static sensors were deployed in our laboratory and their data were observed over the course of four weeks. The static sensors deployed consisted of two door mounted radio-based contact sensors; one which was brand new and one which had been in use for eight years. The rationale for this was to establish if the behaviors of a new sensor varied from those produced by a sensor which had been in use for some time. 
Each of the two contact sensors used returned data to a base receiver, however; they behaved in different manners. The new contact sensor, A, was only capable of sending a single piece of data when any activity occurred about the door. This sensor could not indicate specifically what the event that occurred was. Due to the effects produced by noise, it is possible that in real-world deployment sensor A could send data regardless of the door state. This is a factor which should be considered in the implementation of such a sensor in a role as critical as the monitoring of a door; particularly in a Smart Environment focused on delivering pervasive care to vulnerable occupants.

The older contact sensor, B, sent data to the receiver to indicate specifically if a "door opened" or "door closed" event had occurred. In addition to the contact sensors, two pressure mats were deployed; one was placed inside the door of the laboratory and one was placed outside the door. The pressure mats were deployed in this manner so that both entry and egress could be determined in the environment. The pressure mats in the experimental scenario were set up using the Arduino Uno board. When the pressure mats were not interacted with they returned a consistent reading of 0 . The pressure mats returned a variable reading upon being stepped on. The variance in the readings returned by the pressure mat sensors was not consistent. Upon a user stepping onto the pressure mats, the readings ranged from 50-300. These figures were default values generated by the pressure mats. These were modified using an application written in $\mathrm{C}$ that communicated over the Arduino interface in order to produce a simplified version of the pressure mat data to indicate "presence" or "absence". Due to the basic functionality delivered by both sensor types, they are considered to be "black and white" or binary sensors as they only indicate one of two possible values. A diagram of the static sensor topology is presented in Figure 1.

\subsection{Door State Detection}

The second stage of this approach was to determine the ability of a mobile robot to determine door state without the human in the scenario. This stage focused on the performance of an ultrasonic array placed on the mobile robot. The robot used was the Pioneer 3-DX from Adept Mobile Robots which was equipped with a front facing ultrasonic array that provided $180^{\circ}$ of coverage. Ultrasonic sensors are a largely underused yet cheap robot resource. Due to this, we wished to establish their capabilities in tasks outside of obstacle avoidance. The arrangement of the sensors on the ultrasonic array is shown in Figure 2.

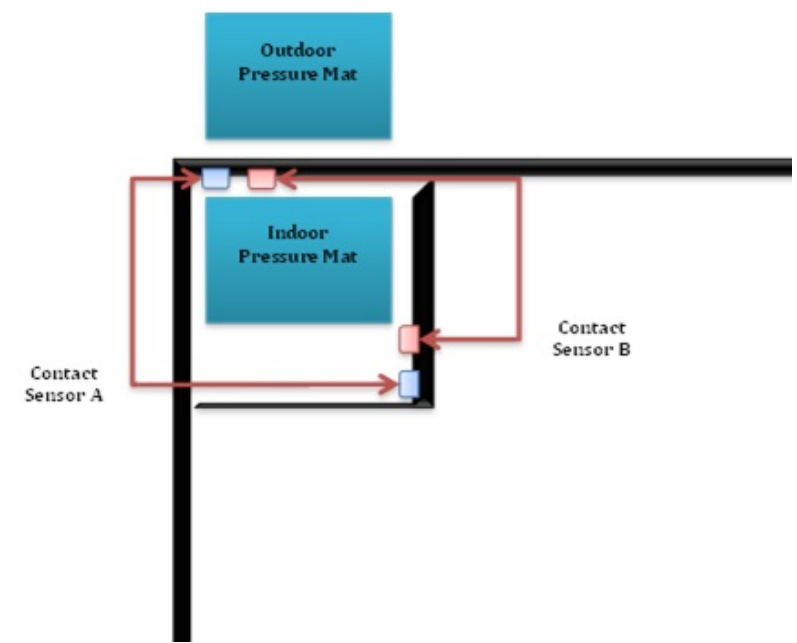

Figure 1: Static sensor topology.

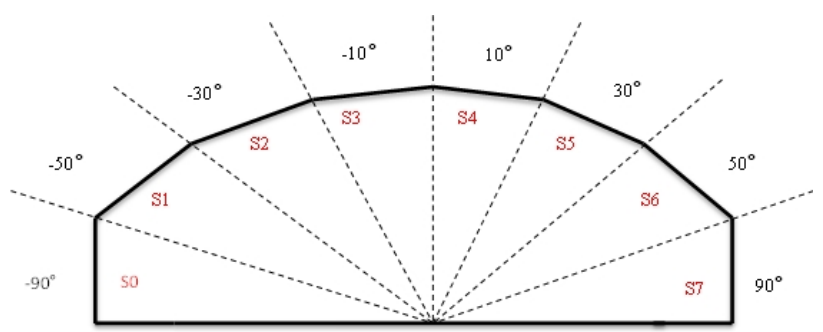

Figure 2: Placement of ultrasonic sensors on Pioneer 3-DX array.

In order to determine this, the door region was divided into six sectors as shown in Figure 3. This robots ultrasonic array was multiplexed; only one disc in the array was active at any time but the transducer fired simultaneously. The array worked by emitting a signal and returning data upon receipt of the echo. The robot was placed at each position about the door in turn. Ten sets of data were obtained for each position at the door. Data collection was carried out with the door closed and no human presence about the door. This was repeated for data collection whilst the door was opened. We also introduced A second robot to the experiment in order to examine its performance in comparison to the 3-DX. The additional robot used was the Dr Robot X80-H. Its experiments were completed in an identical manner.

\subsection{Determining Human Traversal}

The third stage of the experiments investigated if it was possible for a mobile robot to determine the direction of human traversal and thereby infer door state. Informed by the results of the previous experiment, only the $\mathrm{X} 8 \mathrm{O}-\mathrm{H}$ 


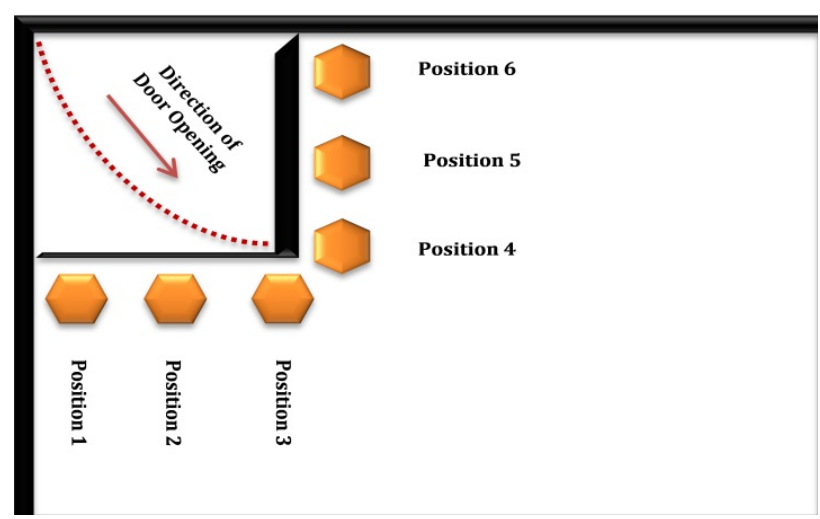

Figure 3: Division of the door region into sectors.

robot was used for this experiment. At this stage the robot was equipped with both ultrasonic and infrared sensors.

The active infrared sensors on the robot worked similarly to ultrasonic; they emitted an infrared ray from their transmitter and then received the return ray with the receiver. One of the limitations of active infrared worth noting is the impact in the accuracy of the readings by variation in the ambient temperature of the environment. As before, ten samples were taken from each robot at each position about the door for both entry and egress.

In addition to the ultrasonic and active infrared sensors, this robot was also equipped with two piezoelectric sensors. Piezoelectric sensors work using passive rather than active infrared. These sensors are popularly used in intruder detection systems. The piezoelectric sensor worked by detecting energy radiation from the human body - it does not emit infrared rays. It is constructed of a pyroelectric element which is covered with a Fresnel lens in order to increase the field of coverage and reduce false positives.

The experiment was carried out using a single test occupant. The occupant was requested to traverse through the door to simulate entry and egress respectively. The experiment was repeated for each of the six robot positions at the door with ten samples of data collected per position.

In the experiments, each robot navigated to a predetermined position in the door region and fed back their data. Upon receipt of the data, a classifier was applied to the data in order to determine the status of activity at the door. The accuracy of each robot at each of the positions designated at the door was measured for evaluation purposes.

\subsection{Introducing Autonomic Management}

In the final stage of this work we designed communicating finite state automata which will be used in future work for the implementation of the autonomic computing paradigm. It is these automata that will introduce adaptivity into the system as a whole. Their design was informed by the results of the preceding experiments.

\section{Results}

\subsection{Sensor Anomaly Types}

Through the observations of the static sensors made prior to commencing the experiments, we were able to determine that two types of sensor anomalies may manifest; systemic anomalies and random anomalies. Systemic anomalies are defined as those which occur as a sensor develops a new behavior over time. In these observations, the systemic anomalies were attributed to the degradation of the sensor battery in contact sensor B. As the battery in the static contact sensor became lower, its behavior changed; instead of sending one sensor event for one realworld event, the sensor began to send multiple events. An example of some of the data gathered is presented in Table 1. Contact sensor A has a Device ID of 40773 while sensor B has a Device ID of 35424. In the data presented in Table 1 we have identified occasions on two different dates on which the erroneous data generated was identical; this was not always the case. This variability resulted in the production of anomalous sensor data from which it is difficult to infer occupant activity.

In order to manage systemic anomalies, we believe that the overall system must use reflective analysis. In the autonomic paradigm, reflective analysis is used to obtain an understanding of new and emergent behavior in a system over time. It is proposed that this reflective analysis can be achieved in the Smart Environment through the deployment of a mobile robot in the role of a watcher; a medium used to monitor and analyze the system over time. The purpose of this role is to obtain an understanding of the manner in which sensors in the environment typically behave and changes thereto in order to assist in introduction of autonomic management by way of adapting to changing behaviors.

Random anomalies are defined as those which occur through sensor noise or the loss of a signal. In our observations, we determined that random anomalies are those which occur when an expected sensor event is, for ex- 
Table 1: Static Sensor Data Showing an Example of a Systemic Anomaly

\begin{tabular}{ccccc}
\hline Device Type & Device ID & Device Status & Time & Date \\
\hline Client Wanderer 1 & 40773 & Alarm & $21: 42: 10$ & $09 / 07 / 14$ \\
Client Wanderer 1 & 35424 & Alarm & $21: 42: 10$ & $09 / 07 / 14$ \\
Client Wanderer 1 & 35424 & Door Closed & $21: 42: 16$ & $09 / 07 / 14$ \\
Client Wanderer 1 & 35424 & Door Closed & $21: 42: 16$ & $09 / 07 / 14$ \\
Client Wanderer 1 & 35424 & Door Closed & $21: 42: 17$ & $09 / 07 / 14$ \\
Client Wanderer 1 & 40773 & Alarm & $09: 13: 25$ & $11 / 07 / 14$ \\
Client Wanderer 1 & 35424 & Alarm & $09: 13: 25$ & $11 / 07 / 14$ \\
Client Wanderer 1 & 35424 & Door Closed & $09: 13: 29$ & $11 / 07 / 14$ \\
Client Wanderer 1 & 35424 & Door Closed & $09: 13: 30$ & $11 / 07 / 14$ \\
Client Wanderer 1 & 35424 & Door Closed & $09: 13: 30$ & $11 / 07 / 14$ \\
\hline
\end{tabular}

ample, not received. For example, the data presented in Table 2 is taken from a test in which the static sensors detected that a person had approached the door from, opened the door and stepped outside but the "door closed" event from contact sensor B was not received despite a real-world door closed event having occurred. In such instances, a mobile robot may be utilized to investigate the door state and determine if the door is in fact still opened. This has the potential to assist in understanding whether the sensor at the door is faulty, or if the door is opened and a care giver should be alerted. By using a mobile robot, the activity at the door can be verified and unnecessary call outs to care givers can be avoided.

\subsection{Door State Detection}

\subsubsection{Pioneer 3-DX}

In our initial experiment with the 3-DX, we analyzed the sensor data obtained from the robot using algorithms within the Weka 3.7 [16] application for feature selection and classification. Data was obtained from the robot for each of the six positions at the door region whilst the door was in the "door closed" and "door opened" state respectively. The results obtained from this study led us to conclude that ultrasonic sensors do provide a viable means of determining door state. The variations in the thresholds of the sensor readings were sufficiently stable which allowed us to reliably detect the door state. However, it was also established that the placement of the mobile robot was a contributing factor in the effectiveness of this approach.

A total of ten samples were taken for each placement of the robot in the door region for each door state. This sample size was selected in order to verify the repeatability of the results. Data collection began immediately after the robot had initially been switched on. Data samples were progressively obtained from the robot as the running time extended. The rationale for this was to examine if variations in the sensor readings occurred based on the length of time for which the robot was running. It became evident during data collection that this was not a factor that needed to be taken into consideration as the sensor readings were consistent regardless of running time.

From the analysis of the sensor data, it was determined that out of the six positions about the door, Position 2 was the position from which the robot provided the most consistent and useful data readings. Whilst in Position 1 the robot was in close proximity to the wall that ran alongside the door region. This caused ultrasonic_0 and ultrasonic_1 to produce readings of 5000 when the door was in the closed state. This issue was investigated further in order to establish if the sensor itself was faulty. We concluded that it was not as the sensor readings changed upon placement in each of the other five positions about the door. It was concluded that the persistence of the reading of 5000 - which is indicative of the absence of a return echo or that an object is closer than 120 millimeters to the array - was a product of the refraction of the ultrasonic echo on the nearby wall. This would compromise the predictability of the "door opened" state as the range of coverage offered by the sensor array would be reduced and the usefulness of the sensor array would diminish. Similarly whilst in Position 6, ultrasonic_6 and ultrasonic_7 behaved in an identical manner as the robot was alongside another wall in the door region. Positions 3, 4 and 5 failed to produce stable sensor readings that could reliably detect the "door closed" event. In addition to the stability of the sensor data results obtained for Position 2, that place- 
Table 2: Static Sensor Data Showing an Example of a Random Anomaly

\begin{tabular}{ccccc}
\hline Device Type & Device ID & Device Status & Time & Date \\
\hline Client Wanderer 1 & 40773 & Alarm & $11: 56: 43$ & $12 / 07 / 14$ \\
Client Wanderer 1 & 35424 & Alarm & $11: 56: 43$ & $12 / 07 / 14$ \\
\hline
\end{tabular}

ment offered a good detection range for the robots sensors along with coverage of the surface area beyond the door region upon opening.

Table 3 contains four samples of the sensor readings obtained for each ultrasonic sensor in the array placed on the robot whilst in Position 2 for both the "door closed" state and "door opened" state. The data obtained from the sensors show that the readings are stable with only minor variation in their values whilst in a given state, yet those values are sufficiently different between states as to clearly depict the "door closed" and "door opened" events separately. The dataset for Position 2 contained 295 instances of the "door opened" event and 265 instances of the "door closed" event. Initially, we applied the Attribute Selection filter which identified the most predictive attributes in the dataset.

In order to quantify the value of the identified attributes, we applied the Ranker algorithm with the Classifier Feature Evaluator using 10 fold cross validation on the dataset. In addition to this, we also used Information Gain Ranking to identify the key sensors for door state determination. The results are presented in Table 4. It is clear from the results that ultrasonic_1, ultrasonic_3, ultrasonic_4, ultrasonic_5 and ultrasonic_6 showed the most significant changes in their data readings between states, yet the thresholds for the sensor values whilst in a given state remained stable.

In order to identify the thresholds that could be used to determine door state, we then classified the dataset for Position 2 using the Least Absolute Deviation tree (LADTree) algorithm based on the most predictive attributes. This algorithm used four of the five most predictive attributes. Each of those chosen had an information gain value of 0.9979. The attribute ultrasonic_3 was excluded as it had a slightly lower information gain value of 0.981 .

From the results of the initial experiment we can conclude that the utilization of an ultrasonic array mounted on a mobile robot is a viable approach to substituting for faulty contact sensors. The door state can be reliably determined due to the significant and stable changes in the readings obtained from the robots ultrasonic sensors.

\subsubsection{Dr Robot X80-H}

Following the results obtained from our initial experiment, we were keen to investigate how well the second robot performed door state detection; particularly whilst in Position 2. We repeated the previous experiment procedure for the X80-H robot; it was placed in each of the six positions at the door. Ten samples of data were recorded per position for each door state. In addition to using the ultrasonic sensors on the X80-H, we also utilized the infrared sensors in order to provide a broader range of coverage as the robot had only three ultrasonic sensors in contrast to the array of eight placed on the 3-DX. The results obtained from this study showed that whilst door state could be reliably detected, the performance of the ultrasonic sensors did not match those of the 3-DX. Rather, the infrared sensors played a prominent role in the door state detection capabilities of this robot. Unfortunately, in investigating the performance of the $\mathrm{X} 80-\mathrm{H}$ whilst placed in Position 2, the results were not as promising as had been hoped.

The dataset for Position 2 was larger for this robot; it contained 512 instances of the "door opened" event and 467 instances of the "door closed" event. The dataset obtained from the robot whilst in Position 2 was analyzed as before; the Attribute Selection filter was applied in order to identify the most predictive attributes. Upon identifying the most predictive attributes we then applied the Ranker algorithm with the Classifier Feature Evaluator using 10 fold cross validation on the dataset. The most surprising result of this was the lack of ultrasonic sensors which featured as predictive attributes - only one ultrasonic sensor was selected. This was surprising as it had been expected that the ultrasonic readings would be more stable than those of the infrared sensors. The results told us that infrared_2, infrared_4, infrared_3 and ultrasonic_3 were the most predictive attributes with ultrasonic_2 ranking fifth with 90.909.

Despite the lack of ultrasonic sensors with a high predictive value, it is clear that door state can also be reliably detected using infrared sensors. We were able to identify the thresholds for state determination by applying the 
Table 3: Sensor Data from 3-DX for Door Opened and Door Closed States in Position 2

\begin{tabular}{ccccccccc}
\hline \multirow{2}{*}{$\begin{array}{c}\text { Door } \\
\text { State }\end{array}$} & \multicolumn{7}{c}{ Ultrasonic sensor array positions } \\
\cline { 2 - 8 } Closed & 478 & 5000 & 991 & 2139 & 851 & 3971 & 1810 & 1439 \\
Closed & 478 & 5000 & 991 & 2139 & 851 & 3973 & 1810 & 1439 \\
Closed & 478 & 5000 & 991 & 2139 & 851 & 3973 & 1810 & 1439 \\
Closed & 478 & 5000 & 991 & 2140 & 851 & 3973 & 1810 & 1439 \\
Opened & 478 & 1662 & 992 & 2570 & 2559 & 2959 & 1265 & 1440 \\
Opened & 478 & 1662 & 992 & 2570 & 2559 & 2959 & 1265 & 1440 \\
Opened & 478 & 1662 & 992 & 2574 & 2559 & 2958 & 1265 & 1440 \\
Opened & 478 & 1662 & 992 & 2574 & 2559 & 2958 & 1265 & 1440 \\
\hline
\end{tabular}

Table 4: Attribute Evaluation and Information Gain Rankings of 3-DX in Position 2

\begin{tabular}{lcccc}
\hline \multirow{2}{*}{ Attributes } & \multicolumn{4}{c}{ Attribute evaluation rankings } \\
\cline { 2 - 5 } & Feature Evaluation & Information Gain & Average Merit & Average Rank \\
\hline ultrasonic_4 & 100 & 0.9979 & $100 \pm 0$ & $1.3 \pm 0.9$ \\
ultrasonic_6 & 100 & 0.9979 & $100 \pm 0$ & $2.1 \pm 0.3$ \\
ultrasonic_5 & 100 & 0.9979 & $100 \pm 0$ & $3.2 \pm 0.6$ \\
ultrasonic_1 & 100 & 0.9979 & $100 \pm 0$ & $3.8 \pm 0.6$ \\
ultrasonic_3 & 99.821 & 0.981 & $99.821 \pm 0.06$ & $4.6 \pm 1.2$ \\
ultrasonic_2 & 56.786 & 0.0144 & $56.786 \pm 0.68$ & $6 \pm 0$ \\
ultrasonic_7 & 55.179 & 0.0396 & $55.179 \pm 0.287$ & $7 \pm 0$ \\
ultrasonic_0 & 52.679 & 0.0178 & $52.679 \pm 0.099$ & $8 \pm 0$ \\
\hline
\end{tabular}

Table 5: Attribute Evaluation and Information Gain Rankings of X80-H in Position 5

\begin{tabular}{ccccc}
\hline \multirow{2}{*}{ Attributes } & \multicolumn{4}{c}{ Attribute evaluation rankings } \\
\cline { 2 - 5 } & Feature Evaluation & Information gain & Average Merit & Average Rank \\
\hline infrared_2 & 100 & 0.99965 & $100 \pm 0$ & $1 \pm 0$ \\
ultrasonic_3 & 100 & 0.99965 & $100 \pm 0$ & $2.4 \pm 1.2$ \\
infrared_3 & 100 & 0.99965 & $100 \pm 0$ & $3.1 \pm 0.3$ \\
infrared_4 & 100 & 0.99965 & $100 \pm 0$ & $4.1 \pm 0.3$ \\
ultrasonic_2 & 100 & 0.99965 & $100 \pm 0$ & $4.7 \pm 0.9$ \\
infrared_1 & 100 & 0.99965 & $100 \pm 0$ & $5.7 \pm 0.9$ \\
infrared_6 & 96.836 & 0.90828 & $96.836 \pm 0.165$ & $7 \pm 0$ \\
infrared_7 & 56.0882 & 0.066 & $56.088 \pm 0.221$ & $8.1 \pm 0.3$ \\
infrared_5 & 55.0336 & 0.00698 & $55.034 \pm 0.572$ & $8.9 \pm 0.3$ \\
ultrasonic_1 & 51.1026 & 0 & $51.103 \pm 0.024$ & $10 \pm 0$
\end{tabular}

LADTree algorithm to the dataset on the most predictive attributes. The algorithm used three of the four most predictive attributes in this case. Each of those chosen had an information gain value of 0.9985 .
Upon examining the data obtained from the remaining positions at the door, Position 3 produced six predictive features. However, two of those attributes did not produce consistently stable results sufficient to differentiate 
between the "door closed" and "door opened" state reliably.

The final position that interested us was Position 5. It became evident that this position was an optimal placement for the X80-H robot in order to determine door state consistently. The dataset for Position 5 produced six predictive features. In this case, two of the most predictive features were ultrasonic sensors with the remaining four being infrared. We repeated the attribute evaluation and ranking stages as before. The results are presented in Table 5 .

In order to identify the sensor thresholds for determining door state, we then classified the dataset for Position 5 with the LADTree algorithm based on the most predictive attributes. This algorithm used four of the six most predictive attributes. Each of those chosen had an information gain value of 0.99965 .

In conclusion, the results tell us that an ultrasonic array with a wide coverage area mounted on a mobile robot can reliably determine door state. The determination of door state is dependent on the robots position and the absence of a human presence in the scenario. It was not anticipated that the infrared sensors would out-perform the ultrasonic sensors in the second experiment. However this has shown that the range of coverage offered by the sensors on a mobile robot operating in an intelligent environment is a strong contributory factor in the performance of door state detection by the robot. Whilst the X80-H performed best in Position 5, the results from the same robot in Position 2 allow us to conclude that this position was still useful for both robots in reliably determining door state. As a result, both Position 2 and Position 5 were selected as points of focus in the final experiment.

\subsection{Determining Human Traversal}

The third experiment in this study focused on the ability of the robot to detect the presence of a human in the door region and determine the manner in which they were traversing the door; entry or egress. We selected the X80-H robot for this experiment. This was due to this robot robots successful performance in the previous experiment using both ultrasonic and infrared sensors.

In this experiment we asked an individual to traverse the door as they normally would. The individual was required to egress and then enter the room. Data was collected for both egress and entry whilst the robot was placed in each of the six positions at the door. Ten samples of data were collected for both egress and entry at each of the six positions. The data obtained for egress and entry were collated for each position at the door and analyzed. The dataset contained two nominal attributes; presence and traversal. The presence attribute depicts whether or not a person has been encountered, the traversal attribute indicates whether entry or egress has occurred.

The Ranker algorithm was applied to each of the collated datasets for each position. We then evaluated the Information Gain Rankings for the attributes and analyzed the data using the LADTree algorithm. The ranking of the attributes was applied twice. Firstly, we ranked the attributes on their ability to determine presence in the door region. These results are presented in Table 6. It is evident that there is a significant reduction in the predictive capabilities of the sensors on the robot when a human is introduced to the scenario. These results deteriorated further upon ranking the attributes for the determination of traversal direction. The results are presented in Table 7.

Whilst the evaluation of the features for the determination of presence in the door region was good, the Information Gain Rankings were not as high as they were when determining door state. Further to this, the Information Gain Rankings for the determination of traversal of the door are almost negligible; there is no single attribute or group of attributes which can provide a clear picture of the direction in which the individual traversed the door.

By analyzing the results using the LADTree algorithm, we were able to obtain some additional information; the presence or absence of a human at the door could be reliably detected. The correctly classified instances of the presence of a person were $99.9536 \%$ of the dataset where the dataset contained 1760 instances of the absence of a person and 393 instances of the presence of a person. In contrast, it was difficult to obtain definitive thresholds for the traversal of the door as the performance of the sensors was low in that respect with high complexity. The correctly classified instances of the traversal of the person through the door only reached $70.9243 \%$ where the dataset contained 1061 instances of entry and 1092 instances of egress. The most accurate classification occurred on the data obtained from the robot whilst it was placed in Position 2. As a result of this, our belief that Position 2 is the optimal placement for a robot to obtain an overview of activity in the door region is supported.

\subsection{Introducing Autonomic Management}

In the final stage of this study we designed communicating state automata for use in our further work on providing autonomic management of anomalous sensor behavior in a Smart Environment. Firstly we created a state machine 
Table 6: Attribute Evaluation and Information Gain Rankings of X80-H in Position 2 Determining Presence

\begin{tabular}{ccccc}
\hline \multirow{2}{*}{ Attributes } & \multicolumn{4}{c}{ Attribute evaluation rankings } \\
\cline { 2 - 5 } & Feature Evaluation & Information gain & Average Merit & Average Rank \\
\hline alarm_1 & 99.953553 & 0.6866 & $0.687 \pm 0$ & $1 \pm 0$ \\
motion_1 & 96.284255 & 0.5514 & $0.551 \pm 0.006$ & $2 \pm 0$ \\
infrared_3 & 84.951231 & 0.1904 & $0.191 \pm 0.007$ & $3.2 \pm 0.4$ \\
infrared_4 & 84.626103 & 0.107 & $0.107 \pm 0.003$ & $8 \pm 0$ \\
ultrasonic_3 & 84.068741 & 0.085 & $0.084 \pm 0.006$ & $9.5 \pm 0.5$ \\
infrared_7 & 83.882954 & 0.0875 & $0.088 \pm 0.004$ & $9.5 \pm 0.5$ \\
motion_2 & 83.046911 & 0.1345 & $0.133 \pm 0.006$ & $7 \pm 0$ \\
alarm_2 & 83.000464 & 0.1523 & $0.153 \pm 0.005$ & $5.7 \pm 0.46$ \\
ultrasonic_2 & 82.721784 & 0.1878 & $0.186 \pm 0.007$ & $3.8 \pm 0.4$ \\
infrared_1 & 82.303762 & 0.0606 & $0.061 \pm 0.002$ & $11 \pm 0$ \\
ultrasonic_1 & 81.978634 & 0.0383 & $0.039 \pm 0.003$ & $12 \pm 0$ \\
infrared_5 & 81.699954 & 0 & $0 \pm 0$ & $14 \pm 0$ \\
infrared_6 & 81.699954 & 0 & $0 \pm 0$ & $15 \pm 0$ \\
traversal & 81.699954 & 0.0148 & $0.015 \pm 0.001$ & $13 \pm 0$ \\
infrared_2 & 81.235485 & 0.1594 & $0.158 \pm 0.007$ & $5.3 \pm 0.46$ \\
\hline
\end{tabular}

Table 7: Attribute Evaluation and Information Gain Rankings of X80-H in Position 2 Determining Traversal

\begin{tabular}{ccccc}
\hline \multirow{2}{*}{ Attributes } & \multicolumn{4}{c}{ Attribute evaluation rankings } \\
\cline { 2 - 5 } & Feature Evaluation & Information gain & Average Merit & Average Rank \\
\hline alarm_2 & 61.4027 & 0.0601 & $0.062 \pm 0.004$ & $1 \pm 0$ \\
infrared_7 & 57.4083 & 0.054 & $0.054 \pm 0.002$ & $2 \pm 0$ \\
ultrasonic_2 & 56.8509 & 0.0231 & $0.023 \pm 0.004$ & $7.9 \pm 1.51$ \\
alarm_1 & 56.2007 & 0.0315 & $0.032 \pm 0.003$ & $4.3 \pm 0.78$ \\
ultrasonic_3 & 56.1078 & 0.0205 & $0.018 \pm 0.006$ & $9.6 \pm 1.28$ \\
infrared_2 & 55.4575 & 0.0299 & $0.03 \pm 0.002$ & $5.1 \pm 0.94$ \\
presence & 55.0395 & 0.0148 & $0.015 \pm 0.002$ & $10.9 \pm 1.22$ \\
ultrasonic_1 & 55.0395 & 0.0192 & $0.017 \pm 0.009$ & $9.6 \pm 2.29$ \\
infrared_3 & 54.3892 & 0 & $0 \pm 0$ & $14.4 \pm 0.49$ \\
infrared_1 & 54.2499 & 0.0401 & $0.04 \pm 0.003$ & $3.1 \pm 0.3$ \\
motion_1 & 52.9958 & 0.0264 & $0.026 \pm 0.003$ & $7.2 \pm 1.17$ \\
motion_2 & 52.9494 & 0.0273 & $0.028 \pm 0.002$ & $6.4 \pm 0.66$ \\
infrared_6 & 51.8346 & 0.0139 & $0.011 \pm 0.006$ & $12 \pm 1.41$ \\
infrared_5 & 50.7199 & 0.0115 & $0.011 \pm 0.001$ & $12.3 \pm 1$ \\
infrared_4 & 50.2555 & 0 & $0 \pm 0$ & $14.2 \pm 0.98$ \\
\hline
\end{tabular}

for the handling of normal static sensor activity. This was called the Normal Activity State Machine (NASM). This machine consists of seven events and eight states. The events in this machine correspond to the "black and white" sensor events generated by the static sensors. It is based on the static sensor topology described in earlier sections. A seventh event was added which allows the machine to be reset to a default state. The states in the machine pertain to the real-world activity that is occurring in the environment. The structure of the NASM is shown in Figure 4.

It was determined that an additional state machine would be required. The second state machine was added 


\begin{tabular}{|c|c|c|c|c|c|c|c|}
\hline Event/State & $\begin{array}{c}\text { E1 IPM } \\
\text { On }\end{array}$ & $\begin{array}{c}\text { E2 IPM } \\
\text { Off }\end{array}$ & $\begin{array}{c}\text { E3 CS } \\
\text { On }\end{array}$ & E4 CS Off & $\begin{array}{c}\text { E5 OPM } \\
\text { On }\end{array}$ & $\begin{array}{c}\text { E6 OPM } \\
\text { Off }\end{array}$ & E7 Reset \\
\hline So Door Closed & S2 & $A 1$ & S1 $A 4$ & $A 2$ & $\mathrm{~S} 3$ & $A 3$ & so \\
\hline S1 Door Open & S5 & $A 1$ & $A 2$ & S0 $A 4$ & S6 & $A 3$ & S0 \\
\hline S2Person@doorinside \& closed & $A 1$ & So & S5 & $A 2$ & S4 & $A 4$ & So \\
\hline S3Person@door outside \& closed & S4 & $A 4$ & S6 & $A 2$ & $A 3$ & So & So \\
\hline S4 Person inside \& outside \& closed & $A 1$ & S3 & S7 & $A 2$ & $A 3$ & S2 & So \\
\hline S5Person@doorinside \& open & $A 1$ & S1 $A 4$ & $A 2$ & S2 & S7 & $A 3$ & So \\
\hline S6Person@door outside \& open & S7 & $A 1$ & $A 2$ & S3 & $A 3$ & S1 & So \\
\hline S7 Person inside \& outside \& open & $A 1$ & S6 & $A 2$ & S4 & $A 3$ & S5 & So \\
\hline
\end{tabular}

Figure 4: The structure of the NASM developed.

in order to address the detection of an anomalous sensor event and the investigation of that event by the mobile robot. This was the Error Handling State Machine (EHSM). Upon the detection of an anomalous sensor event, the actions in the NASM generated an event into the EHSM as shown in Figure 5. The EHSM must then communicate with the mobile robot operating in the environment in order to deliver instructions as to what sensor it should investigate.

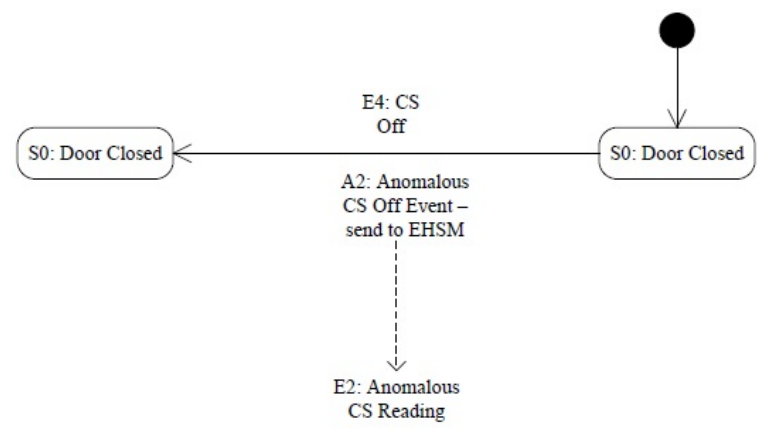

Figure 5: An example of the logical flow between the NASM and EHSM.

We then pursued the introduction of adaptivity into the NASM. Once the outcome of the investigation of the anomalous sensor has been completed, the EHSM must then communicate with the NASM. That communication may take one of two forms; instruct the NASM to adapt its policy in relation to a particular sensor or generate the Reset event into the NASM to return it to its default state.
For example, if it is discovered that a static sensor has developed a behavior whereby it generates two contact sensor events for one real-world event, the EHSM will instruct the NASM that it must adapt its policy to wait for two contact sensor events instead of one. In this manner the presence of such behavior in future is handled without the need to throw an error. It is this approach that is proposed for the introduction of self-management to the environment through self-investigation, self-configuration and self-healing.

\section{Discussion}

Through the deployment of static sensors in a Smart Environment it is possible to use the data generated to determine the activities undertaken by the occupant. It is through the analysis of this data that assistance can be delivered; particularly upon the identification of unusual sensor activity which may be indicative of a problem. There are several forms that the assistance provided may take. Those vary from contacting a carer if a fall is detected to providing a reminder if the occupant has failed to take their medication. Whilst effective means of delivering intervention upon unusual occupant activity detection have been developed, there remains a critical need to address how the technology itself is monitored.

In our work we have established through observations of static environment sensors that two types of sensor anomalies may arise; random anomalies and systemic 
anomalies. Random anomalies are those which occur sporadically. The cause of such anomalies may arise from sensor noise or sporadic fluctuations due to ambient temperature or signal disturbance. Systemic anomalies have been classed as those which arise as the behavior of a sensor changes over time. The cause of these changes in behavior may be attributed to hardware degradation of the battery, board or other physical component. The research presented in [17] investigates the prevalence of sensor faults in real-world datasets. These faults are detected using four popular approaches to fault detection methods broadly used in wireless sensor networks; rulebased, estimation, time-series analysis and learning methods. The authors highlight that the effectiveness of each of these methods varies greatly in their application to a realworld dataset. Consequently the authors identify a need for a more generic approach to fault detection to be developed which would produce a higher belief-confidence than those tested.

In our research, the detection of faults is encompassed in the investigation of anomalous sensor behaviors. This is implemented through the use of communicating adaptive state automata. We believe that this is a more lightweight approach that is more easily scaled than onerous rulebases or time-series based analyses. In our research, the detection and investigation of anomalous sensor behaviors occurs in real-time. The presence of a fault is confirmed through the comparison of the robots data with that of the static sensor data. The communicating state automata can then determine the action the system should take. Additionally, having added adaptivity to the state automata, adaptation to new and emergent behavior is easily facilitated through the generation of new events, states or actions in the state automata as required.

The approach proposed in [18] focuses on the assessment of risks in a Smart Environment rather than the examination of anomalous sensor behaviors. The approach is based upon a multi-agent system. A number of sensors are treated as agents in the system. Those agents use their data to assess the risk of a given situation. This work focuses particularly on those with mild dementia. Though no practical implementation has been reported of this approach, theoretically an alarm would be sounded when a situation deemed to be a high risk has been detected; such as the presence of high levels of gas in the environment. Whilst this work is presented as proactive, there are no measures taken to verify the correctness of the systems conclusion and the assessment of risk is based on data held in a knowledge base. We believe that this is not a reliable approach as the behavior of individuals is highly variable. The use of a knowledge base would require extensive maintenance and updating. In our work we utilize the mobile robot as a means of verifying anomalous sensor behavior before then sending an alert to a carer or engineer. By verifying anomalous sensor behavior, the reporting of false alarms can be reduced and the need for human intervention - which are set to become more limited resources in the care delivery context - is limited to those situations which the system cannot physically cater for itself such as the replacement of a sensor node.

In [19] the authors argue that environments used for the delivery of AAL should be considered as safety-critical systems due to the impact that the failure of the system can have on the quality of human life. They also highlight that dependability is a key system requirements in the AAL setting which has not, thus far, been sufficiently addressed prior to implementation. In order to address this, the authors propose detailed approaches to software modeling prior to physical deployment of the technology. Whilst the authors briefly consider the consequences of hardware faults and failures in the environment, an approach to handling these is not proposed but rather it is suggested that software dependability is first ensured. We would strongly agree with this stance; particularly in the application of the AAAL concept: accurate system modeling is crucial to ensure the reliable operation of the system. However despite the most dependable of software, it is an inherent characteristic of hardware that at some point in time it can and will fail. We address both the software and hardware limitations on Smart Environment technology through the use of self-managing software incorporated with assistive robots deployed in the environment which are capable of addressing hardware faults through substitution.

The work presented in [20] presents a pilot study on the use of a socially assistive robot for the delivery of therapy to a dementia sufferer. The robot consists of a human torso style design mounted on a Pioneer 2-DX. The ultrasonic array on the 2-DX is identical to that on the 3-DX. This pilot study would be an example of the type of assistive robots that may perform the additional task of addressing anomalous sensor behaviors in the environment, particularly given that the robot would not be interacting with the human on a continuous basis throughout the day. The results of the sensor substitution capabilities that we have described in our paper show that the approach could also be applied to the 2-DX model described in [20]. The performance of the robot in the substitution and anomaly verification role could be improved through the addition of infrared and piezoelectric sensors for the detection of the human in the scenario. This approach has the potential to be extended to include the provision of reminders to the 
occupant to close a door if, for instance, the robot has determined that the occupant has left the door lying opened.

The research presented in [21] makes the case for the need to develop systems which deliver ambient intelligence with consideration to non-functional software requirements; particularly adaptivity. Additional requirements that the system should meet include robustness, availability and resource efficiency. The autonomic computing paradigm is a means of delivering those criteria. In our work the adaptive state automata we have designed are capable of self-CHOP, though the practical deployment will form part of our future work. We believe that AAAL is an important step in the future of assisted living technologies. The introduction of self-management has the potential to facilitate the requirements identified in [21]. Indeed current applications of the autonomic paradigm are minimal; this technology is typically employed in other safetycritical systems such as the space industry.

The current literature that exists in the areas of assisted living, assistive robotics and Smart Environments do not currently employ the approach we have proposed to the management of the technology in a Smart Environment. We have identified the need for self-management in this domain. We suggest that utilizing existing assistive robot technologies in these environments is an effective way to move towards the self-management of anomalous sensor events. This increases the need for the robots integration into the environment and provides an additional function that may be made applicable to a wide range of assistive robots. There is further potential for this premise to be extended into investigating occupant activities that are deemed to be unusual. We have shown in this work that mobile robots are capable of providing sensing substitution in the environment and of informing the performance of reflective analysis by the system as a whole over time. The creation of a self-managing AAAL environment requires liaison between the design of the systems software and the incorporation of the elements that exist within the environment so that cooperative peer-to-peer communication can be implemented in order to allow the system to manage its own behaviors. In this manner, the need for human intervention is minimized to occasions on which the system cannot perform a physical task or a problem has been detected pertaining to the environment occupant.

\section{Conclusion}

In this research we proposed that mobile robots in Smart Environments can facilitate the self-healing and self- configuration of the system in the face of anomalous sensor behaviors through the investigation of anomalous sensor behaviors and the substitution of sensor faults. This is supported through the design of communicating state automata which handle communications between the sensing components of the environment. The results of the experiments completed within this research have shown that the use of a mobile robot is a viable approach to the substitution of sensing functionality about a door in a Smart Environment.

One of the limitations of this work that we have identified is the time lapse that exists between the occurrence of anomalous sensor behavior and the robots navigation to the site of the sensor in order to investigate or perform substitution as required. However, despite this shortcoming, the deployment of the robot is a useful approach for reflective observation of the behavior of sensors in the environment in order to adapt the systems policies in accordance with new or emergent sensor behavior. The effectiveness of this approach is influenced by robot placement and the distribution of sensors on-board the robot.

We believe that this approach shows promise in providing the self-management of Smart Environment technology whilst also introducing a new role to the assistive robot functioning in the environment. In our future work we will focus on the development of the state automata created based on this research in order to further develop their capacity for adaptability and scalability. We will also investigate an approach to address the time lapse that exists between anomalous behavior detection and robot deployment.

Acknowledgement: This work is funded by the Northern Ireland Department for Employment and Learning (DEL http://www.delni.gov.uk). This research has additionally been awarded the 2012 Annual Research Bursary of the Health Informatics Society of Ireland (HISI http://www. hisi.ie/).

\section{References}

[1] World Health Organisation, Ageing and life course, 2011

[2] The Institute for Fiscal Studies, Pressures in UK healthcare: Challenges for the NHS., 2000

[3] J.M. Robine, J.P. Michel, F.R. Hermann, (2007) Who will care for the oldest people in our ageing society? The BMJ, 2007(334), 570

[4] I. Korhonen, J.E. Bardram, Guest editorial introduction to the special section on pervasive healthcare, IEEE Transactions on Information Technology in Biomedicine, 8(2004), 229-234

[5] Ambient Assisted Living Joint Programme, Objectives, 2007 
[6] U. Varshney, Pervasive healthcare, Computer, 36(2003), 138 140

[7] R.R. Murphy, Introduction to Al robotics, Cambridge, Massachusetts, London, England, MIT Press, 2000

[8] M. Pollack, S. Engberg, J.T. Matthews, S. Thrun, L. Brown, D. Colbry, C. Orosz, B. Peintner, S. Ramakrishnan, J. Dunbar-Jacob, C. McCarthy, M. Montemerlo, J. Pineau, N. Roy, Pearl: A mobile robotic assistant for the elderly, Proceedings of Workshop on Automation as Caregiver: the Role of Intelligent Technology in Elder Care (2007), 2007

[9] C.K.Y. Lai, D.G. Arthur, Wandering behaviour in people with dementia, Journal of Advanced Nursing, 44(2003), 173-182

[10] R. Preece, (2012, Oct. 7). Dementia sufferer died after being locked out of care home when he was able to wander outside in middle of the night, [Online]. Available: http://www.dailymail.co.uk

[11] S. Davies. (2013, November 4). Wife's anger after Southampton care home unaware dementia patient has walked out, [Online]. Available: http://www.dailyecho.co.uk

[12] CTV British Columbia. (2013, December 9). Family wants answers after senior leaves care home, dies outside, [Online]. Available: http://bc.ctvnews.ca/family-wants-answersafter-senior-leaves-care-home-dies-outside-1.1582407

[13] R. Sterritt, C. Nugent, Autonomic computing and ambient assisted living - extended abstract, IEEE International Conference and Workshops on Engineering of Autonomic and Autonomous Systems, (2010), 2010, 149-151

[14] P. Horn, Autonomic computing: IBM perspective on the state of information technology, 2001
[15] R. Sterritt and D. Bustard, Towards an autonomic computing environment, Database and Expert Systems Applications (2003), 2003, 694-698.

[16] M. Hall, E. Frank, G. Holmes, B. Pfahringer, P. Reutemanm and I.H. Witten, The WEKA data mining software: an update, SIGKDD Explorations, 11(2009)

[17] A. B. Sharma, L. Golubchik, R. Govindan, Sensor faults: detection methods and prevalence in real-world datasets, ACM Transactions on Sensor Networks, 6(2010), 1-39

[18] S. Nefti, U. Manzoor, S. Manzoor, Cognitive agent based intelligent warning system to monitor patients suffering from dementia using ambient assisted living, International Conference on Information Society, (2010), 2010, 92-97

[19] G. N. Rodrigues, V. Alves, R. Silveira, L. A. Laranjeira, Dependability analysis in the Ambient Assisted Living Domain: An exploratory case study, Journal of Systems Software, 85(2012), 112-131

[20] A. Tapus, C. Tapus, M. J. Mataric, The use of socially assistive robots in the design of intelligent cognitive therapies for people with dementia, IEEE International Conference on Rehabilitation Robotics (2009), 2009, 924-929

[21] J. Nehmer, M. Becker, A. Karshmer, R. Lamm, Living assistance systems: An ambient intelligence approach, Proceedings of the 28th International Conference on Software Engineering (2006, Shanghai, China), 2006, 43-50 